\title{
Renal transplantation in patients with HIV
}

\author{
Lynda A. Frassetto, Clara Tan-Tam, and Peter G. Stock \\ Departments of Medicine and Clinical Research Center (LA Frassetto) and of Surgery (C Tan- \\ Tam, PG Stock), University of California San Francisco, CA, US
}

\section{Abstract}

HIV infection has been a major global health problem for almost three decades. With the introduction of highly active anti-retroviral therapy in 1996, and the advent of effective prophylaxis and management of opportunistic infections, AIDS mortality has decreased markedly. In developed countries, this once fatal infection is now being treated as a chronic condition. As a result, rate of morbidity and mortality from other medical conditions leading to end-stage liver, kidney and heart disease is steadily increasing in individuals with HIV. Presence of HIV infection used to be viewed as a contraindication to transplantation for multiple reasons:,concerns for exacerbation of an already immunocompromised state by administration of additional immunosuppressants; the use of a limited supply of donor organs with unknown long-term outcomes; and, the risk of viral transmission to the surgical and medical staff. This Review examines open questions on kidney transplantation in patients infected with HIV-1 and clinical strategies that have resulted in good outcomes. It also describes the clinical concerns associated with the treatment of renal transplant recipients with HIV.

\section{Introduction}

Since the early years of the AIDS epidemic, the medical and scientific community has been aware of various renal diseases that develop in patients with HIV. ${ }^{1,2}$ Renal diseases directly related to HIV infection include HIV-associated nephropathy (HIVAN), immune complex diseases, and thrombotic microangiopathy. Although the widespread used of highly active antiretroviral therapy (HAART) has decreased the incidence of HIV-related renal disease, ${ }^{3}$ the overall prevalence of renal disease continues to increase among patients with HIV. ${ }^{4,5}$ Potential explanations include inadequate HAART, drug toxicity, increased survival rates leading to an increase in the proportion of elderly patients, and chronic viral co-infections (i.e. viral hepatitis).. 6,7

The most aggressive HIV-related renal disease is HIVAN, which occurs in approximately $10 \%$ of patients with HIV. ${ }^{8}$ These patients can progress to end-stage renal disease (ESRD) within weeks to months. Although the etiology of HIVAN is not completely understood, direct infection of HIV-1 of renal epithelial cells is associated with the onset of the disease. ${ }^{9}$ This variant of focal sclerosing glomerulonephritis is diagnosed by kidney biopsy and afflicts mainly patients of sub-Saharan African descent. ${ }^{10,11}$ HIVAN is currently the third most common etiology of ESRD among African Americans aged 20-64 years after diabetes and hypertension. ${ }^{12,13}$ Co-infection with hepatitis B virus (HBV) or hepatitis C virus (HCV) is also common, and affected patients are at risk of developing viral hepatitis-associated

\footnotetext{
Correspondence: L Frassetto, UCSF campus box 0126, room M1202, San Francisco, CA 94143, US frassett@gcrc.ucsf.edu. Competing interests

The authors declare no competing interests.
} 
glomerulonephritis. Nearly $1 \%$ of all patients with ESRD in the US and Europe are estimated to have HIV. 5,14

Nephrotoxicity could be induced or exacerbated by anti-retroviral medications, such as ritonavir, as well as infection prophylaxis medications such as trimethoprim sulfamethoxazole. Some drugs routinely administered to patients with HIV (i.e. indinavir, atazanavir, sulfadiazine, ciprofloxacin and intravenous aciclovir) can also precipitate in the renal tubular lumen, thereby causing renal failure. ${ }^{7,15}$ Tenofovir and adefovir, which are HAART medications also used in the management of lamivudine-resistant HBV, are potentially nephrotoxic agents. Calcineurin inhibitors (CNIs) used in immunosuppressive therapy, such as ciclosporin and tacrolimus, are nephrotoxic, both directly and, by causing vasoconstriction and increasing blood pressure, indirectly. The toxicity of CNIs can be exacerbated by inhibition of the cytochrome P450 system by other HAART agents such as protease inhibitors (PIs) and agents commonly used for fungal prophylaxis and treatment, such as fluconazole. In addition, some HAART agents can induce insulin resistance, diabetes mellitus, hypertension and hyperlipidemia, ${ }^{16}$ all of which are major risk factors for ESRD in the US.

Notably, however, survival of patients on dialysis who have HIV has improved in patients whose disease is under control on HAART (e.g., those with high CD4 ${ }^{+}$T-cell counts) and is now similar to that of patients who are not infected with HIV. ${ }^{17,18}$ Kidney transplantation in patients without HIV is known to result in better survival rates than continuing dialysis. ${ }^{19}$ Given the advances in treatment of HIV over the past decades, investigating the safety and efficacy of renal transplantation in patients with HIV has become a research priority.

\section{Early outcomes of transplantation}

The first prospective study performed in the HAART era followed 10 patients with kidney transplants who had not undergone induction therapy and whose maintenance therapy included ciclosporin and mycophenolate mofetil (MMF). These patients had been selected for transplantation because they had no history of opportunistic infections and HIV virus was undetectable. This study demonstrated similar one-year patient and graft survival rates to patients without $\mathrm{HIV} ;{ }^{20}$ however, more than half the study participants experienced acute rejection, which required aggressive treatment with an anti-lymphocyte globulin. ${ }^{20,21}$

Subsequent retrospective analyses, case reports and small prospective studies include a study of the outcomes in 63 patients with HIV with deceased-donor-kidney-transplants and 37 with living-donor-kidney transplants from the US Scientific Registry of Transplant Recipients, ${ }^{22}$ a report of 47 patients with HIV in the US Kidney Data System, ${ }^{23}$ and a review of 18 patients transplanted at four US transplant center through $2003 .{ }^{24}$ Early results from these studies indicate that patient and graft outcomes are similar to those reported for patients without HIV, although allograft rejection rates are higher. Roland et al. ${ }^{25}$ prospectively followed 18 kidney transplant recipients with HIV for over 3 years and observed that the incidence of rejection episodes at 1-year and 3-years was 52\% and 70\%, respectively. In spite of the high rejection rates, however, patient and graft survival rates were $94 \%$ and $83 \%$ at 3 years. Other encouraging reports demonstrate comparable patient and graft survival rates in patients with and without HIV. ${ }^{26,27,28,29}$

Rates of acute rejection of renal transplants in patients with HIV range from 13\% to 67\%. 20,21,26-29 Protocol biopsies at one institution also demonstrated subacute rejection rates of $29 \% .{ }^{31}$ In addition, numerous studies have reported a higher acute rejection rate in patients of sub-Saharan African descent than in those from other ethnic backgrounds. ${ }^{20,21,28,29}$ The etiology of such high rejection rates is unclear, although dysregulation of the immune system or insufficient immunosuppression are two possible causes. Data from a prospective 
study by Kumar et al. ${ }^{31}$ demonstrated that induction therapy by anti-CD25 antibody administration and maintenance therapy with sirolimus resulted in a decreased rejection rate; however, the 1-year patient and graft survival rates were similar to those in other high risk populations, $85 \%$ and $75 \%$, respectively. Other encouraging preliminary data come from a small retrospective study that examined outcomes in eight renal allograft recipients with HIV. Study participants had undergone induction therapy with an anti-interleukin-2 receptor antibody and maintenance therapy with ciclosporin, MMF and prednisone. At a median follow-up of 15 months, patient and graft survival rates were $100 \%$ and $88 \%$, respectively, and the rate of acute rejection was $13 \% .{ }^{29}$ In both of these studies, the patients did not show any HIV infection progression, although results require further verification with longer follow-up and larger cohort of patients than those examined.

Despite high rates of acute rejection, kidney transplantation has proven a successful renal replacement treatment modality for patients with HIV. Although patient and graft survival rates at three years are comparable with those observed in patients without HIV, the high number of early rejection episodes might have a negative effect on the long-term graft function of patients with HIV. Reducing rejection rates, therefore, will be a primary goal in future research, and collaborative research efforts are currently underway to investigate the etiology of acute rejection in these patients.

\section{Patient selection criteria}

The criteria that patients with HIV must satisfy to be eligible for transplantation continue to evolve as experience in managing transplant recipients with HIV accumulates. Traditionally, selection criteria were predicated on the concern that immunosuppressing patients with HIV would accelerate progression of HIV to AIDS. This effect on progression has however not materialized in practice, and the selection criteria for suitable allograft recipients are progressively being liberalized. Potential eligibility requirements for solid organ transplantation in patients with HIV are based on the North American and European transplantation criteria for patients without HIV (see Table 1). Mandated requisites include a prolonged period of abstinence from alcohol and narcotics, and demonstration of social support. Eligibility criteria specific to patients with HIV include defined minimum levels of $\mathrm{CD} 4^{+} \mathrm{T}$-cell counts and nondetectable levels of HIV viral load, absence of specific patterns of HIV viral resistance and absence of history of or ongoing specific opportunistic infections. ${ }^{30,31}$ Most transplantation centers require recipients to have a CD4 ${ }^{+} \mathrm{T}$-cell count greater than 200 cells $/ \mathrm{ml} .{ }^{32}$ Given that in children, the percentage of $\mathrm{CD} 4^{+} \mathrm{T}$-cell is a better reflection of an intact immune system, ${ }^{33}$ pediatric patients $1-2$ years of age or 2-10 years of age, the requirements in the US NIH multicenter trial require a $\mathrm{CD}^{+} \mathrm{T}$-cell percentage greater than $30 \%$ and $20 \%$, respectively. Evidence also suggests that the ratio of $\mathrm{CD}^{+}$to $\mathrm{CD} 8^{+} \mathrm{T}$-cells could be used as a surrogate of HIV-1 infection in pediatric patients. ${ }^{34}$

A history of opportunistic infections is no longer considered an automatic exclusion criterion in most European countries and North America, as long as the opportunistic infections can be treated successfully in immunosuppressed patients. In the NIH multicenter prospective trial, opportunistic infections which remain a contraindication for solid organ transplantation in general include chronic cryptosporidiosis, progressive multifocal leukoencephalopathy, and systemic Kaposi's sarcoma, whereas a history of tissue-invasive cytomegalovirus infection is no longer considered a contraindication given the availability of effective therapies. Consideration for transplantation of patients with resolved visceral Kaposi's sarcoma is based on the efficacy of sirolimus - a commonly used immunosuppressive agent—in treating the sarcoma. ${ }^{35,36,37}$ 
A clinical challenge in managing kidney transplant recipients with HIV is the relatively high frequency of co-infection with HBV and HCV. Approximately $30 \%$ of patients with HIV have hepatitis $\mathrm{C}$ co-infection ${ }^{38}$ and approximately $10 \%$ hepatitis B co-infection. ${ }^{39} \mathrm{In}$ patients with either co-infection, the extent of liver damage needs to be assessed before consideration for kidney transplantation. Management strategies for patients with HIV who have minimal liver disease but have HBV or HCV infection can be challenging (see below). However, data suggest that treatment with HAART or MMF might help slow progression of liver disease ${ }^{40}$ and improve survival rates after renal transplantation. ${ }^{41}$

\section{Donor selection criteria}

A major source of concern in renal transplantation is that the duration of time spent on the transplantation waiting list continues to increase as demand for organs soars while the supply of deceased-donor organs remains stable. One study of the United Network for Organ Sharing (UNOS) database demonstrated that lower death-censored graft survival at one year in patients with HIV and renal transplants from deceased donors older than 50 years of age and with cold ischemia time longer than 16 hours. ${ }^{42}$ One strategy to address these problems is to use organs from living donors. Another is to use kidneys from 'infectious-high-risk' deceased donors-individuals who tested negative for HIV, HBV and $\mathrm{HCV}$, but, based on social history, could have acquired the infections shortly before becoming kidney donors. Many recipients of renal transplants with HIV received organs from these donors. The use of extended-criteria deceased donors is appropriate for elderly patients with HIV (using the same recipient criteria as those for patients without HIV). Currently, the use of pediatric en-bloc kidneys is not recommended because of the high risk of rejection and the possibility that these small kidneys might not tolerate this rejection insult.

\section{Strategies for immunosuppression}

As mentioned, the reasonable belief that immunosuppression could result in progression of HIV disease has in the past discouraged transplantation in people with HIV. However, many agents currently used for post-transplantation maintenance immunosuppression (e.g. MMF, ciclosporin, tacrolimus, and sirolimus) have anti-retroviral properties. MMF virostatic action is thought to result from the depletion it causes of guanoside nucleosides, which are necessary for the virus lifecycle. ${ }^{43,44}$ Ciclosporin and tacrolimus have well-documented antiretroviral effects through selective inhibition of infected cell growth ${ }^{45,46}$. These agents interfere with HIV pathogenic protein functions, which ultimately results in the reduction of virus formation..$^{47}$ Ciclosporin and tacrolimus can however cause glucose intolerance, which can be exacerbated by administration of sirolimus.

Since many patients with renal allografts, particularly those with HIV, experience some degree of renal insufficiency, sirolimus, an inhibitor of the mammalian target of rapamycin and an anti-proliferative agent, has been considered as an alternative to CNIs. Similarly to CNIs, sirolimus also exerts some antiretroviral activity through suppression of T-cell activation, suppression of professional antigen presenting cell function, and disruption of infective virion replication ${ }^{34,35}$ Sirolimus also decreases the expression of C-C chemokine receptor type 5 on monocytes and lymphocytes, thus potentially preventing the HIV virus from entering these cells and replicating. ${ }^{36}$

In the initial clinical trials of organ transplantation in patients with $\mathrm{HIV},{ }^{20,21}$ immunosuppressive regimens focused on maintenance therapy with agents with known antiretroviral qualities. This therapy consisted of a combination of steroids, a CNI and MMF. However, organ recipients with HIV can mount an alloimmune response ${ }^{20,21}$ and renal transplant recipients with HIV have a higher rejection rate than their counterparts without 
HIV. ${ }^{20,21}$ For this reason, induction therapy with interleukin-2 receptor inhibitor has been introduced. ${ }^{26,27}$ Whether induction with even more potent immunosuppressants than interleukin-2 receptor inhibitor will be required is unknown. Nonetheless, most transplantation centers are reluctant to utilize lymphocyte depleting agents for induction, as these agents severely deplete $\mathrm{CD} 4^{+} \mathrm{T}$-cells for several months. Nonetheless, these these potentdepleting agents have successfully reversed aggressive rejection in several patients. ${ }^{21}$

\section{Drug interactions}

In most centers, allograft recipients with HIV receive the same HAART regimens they received before transplantation. Early studies demonstrate that using this strategy HIV does not progress to AIDS. ${ }^{20,25,27}$ If, for any reason, patients are unable to take one or more of their HAART drugs, HAART should be discontinued immediately to avoid development of drug-resistant HIV strains. Initial experience suggests that transplant recipients with HIV can tolerate HAART withdrawal for several weeks without changes in viral load and CD4 ${ }^{+}$ T-cell counts. ${ }^{20,48}$

Management of solid organ transplantation in patients with HIV is complicated by multiple drug interactions between HAART agents and immunosuppressants. The most notable drug interaction occurs between antiretroviral medications and immunosuppressive agents that induce or inhibit the P-glycoprotein efflux transporters and cytochrome p450 3A (CYP3A4) metabolizing enzymes found in the gut and liver. These interactions can lead to unexpected increases or decreases in drug plasma levels, and result intoxic side-effects, organ rejection or HIV disease breakthrough. CNIs, such as ciclosporin, and PIs inhibit both P-glycoprotein and CYP3A4 activity. This results in increased levels of circulating drugs due to an increase in cross-membrane drug transport and a decrease in drug metabolism, respectively. Nonnucleoside reverse transcriptase inhibitors (NNRTIs), such as efavirenz, can markedly induce CYP3A4 activity increasing drug metabolism and leading to decreased plasma drug levels. ${ }^{49,50}$

These pharmacologic interactions are well documented in a study describing the pharmacokinetics and dosing modifications of ciclosporin, sirolimus and tacrolimus in 35 liver or kidney transplant recipients on NNRTIs, PIs or both. ${ }^{51}$ Patients on PIs and ciclosporin required only $20 \%$ of the immunosuppressant dose administered to renal transplant recipients without HIV. Low doses of ritonavir, a potent inhibitor of both Pglycoprotein and CYP3A4, is often used to increase or "boost" other PI levels. Patients on a ritonavir-boosted PI regimen required even lower doses of immunosuppressants than patients on other HAART regimens. In patients on tacrolimus or sirolimus, not only was the immunosuppressant dose markedly decreased, but the dosing interval increased more than five-fold. Similar findings have been demonstrated by other investigators in liver transplant recipients. ${ }^{52,53,54,55}$ Notably, azole antifungals and macrolide antibiotics also inhibit the CYP3A4 system. ${ }^{56}$

Additional potential interactions between HAART and immunosuppression regimens should be kept in mind. Patients taking steroids are usually taking proton pump inhibitors, which can reduce intestinal absorption of the PI atazanavir and, thus, the drug's plasma concentration..$^{57,58}$ Patients on proton pump inhibitors and atazanavir require, therefore, PI treatment to be boosted by the administration of ritonavir. ${ }^{59}$ In vitro evidence suggests that MMF is antagonistic to the anti-HIV replication effects of zidovudine and stavudine ${ }^{53}$, so these drug combinations should probably be avoided. Zidovudine is also the only myelotoxic HAART agent, and when combined with another myelotoxic agent such as MMF could have additive myelosuppressive effects. 


\section{Management of comorbities}

\section{HBV}

HBV resistance to lamivudine is common in kidney transplant recipients who have been on lamivudine-containing HAART regimens. These patients require therapy with tenofovir or other appropriate antivirals. Containment of lamivudine-resistant hepatitis B posttransplantation in kidney recipients with HIV and HBV has not been problematic with the increasing number of anti-HBV agents available.

\section{HCV}

Post-transplantation immunosuppression exacerbates hepatitis $\mathrm{C}$ infection in kidney allograft recipients, which makes the management of patients with HIV-HCV co-infection particularly challenging. Clearance of $\mathrm{HCV}$ with interferon ribaviron therapy should be attempted before transplantation. Although efficacy of this approach is poor ${ }^{60}$ (particularly in patients infected with the most common phenotype of the $\mathrm{HCV}$ virus, genotype 1) this attempt is worthwhile, since $\mathrm{HCV}$ clearance post-transplantation with interferon therapy increases the risk of organ rejection in a recipient population that is already particularly subject to this outcome. ${ }^{48,61}$ Early data on kidney transplantation in patients with HIV and HCV have shown acceptable outcomes, but the long-term influence on rates of rejection and progression of $\mathrm{HCV}$ are being closely monitored in the NIH multicenter prospective trial ${ }^{62}$

\section{Bone metabolism disorders}

Renal transplant recipients with HIV are at particularly high risk of low bone mineral density and abnormal bone metabolism. ${ }^{63,64}$ These abnormalities are secondary to multiple factors, including: renal-failure-associated hyperparathyroidism leading to osteitis fibrosa cystica; low vitamin D levels leading to low bone turnover; metabolic acidosis and reduced patient physical activity leading to osteoporosis; administration of post-transplantation medications such as steroids ; HIV-associated low androgen levels in both men and women; 65,66 and administration of antiretrovirals, such as tenofovir and didanosine, which have been associated with reduced bone mass. ${ }^{67}$ Dual-energy X-ray absorptiometry data from the University of California San Francisco (unpublished data from the NIH trial) shows low bone mass density both before and after transplantation. If tolerated, hormone therapy or administration of bisphosphonates can be implemented to try and restore bone mass levels.

\section{Managing risk of comorbidities}

\section{Prophylaxis to prevent co-infections}

Renal transplant recipients with HIV should receive the standard post-transplantation prophylaxis regimens that are used in all transplant recipients. These regimens include prophylaxis for Cytomegalovirus, fungal infections, and Pneumocystis carinii pneumonia during the early post-operative period. If patients receive treatment with lymphocytedepleting regimens for management of rejection, the prophylaxis regimens should be resumed for 3-6 months after discontinuation of the anti-rejection treatment (See Table 2). 21,62 For patients with HIV, lifelong prophylaxis with trimethoprim-sulfamethoxazole for Pneumocystis carinii pneumonia is recommended by most transplantation centers. For patients allergic to sulfonamides, dapsone or atovaquone are viable alternative options. For patients with $\mathrm{CD}^{+}{ }^{+} \mathrm{T}$-cell counts under 75 cells $/ \mathrm{ml}$, prophylaxis for Mycobacterium avium complex is also recommended until the $\mathrm{CD}^{+} \mathrm{T}$-cell counts rebound. Transplant recipients with HIV should also receive vaccinations according to HIV guidelines. ${ }^{68}$ 


\section{Cardiovascular diseases}

Individuals with HIV are at high risk of cardiovascular diseases. Individuals with ESRD often have high blood pressure, even if that was not the etiology of their renal failure. ${ }^{69}$ PIs disturb lipid metabolism, an effect that leads to high levels of cholesterol and triglyceride and causes insulin resistance. ${ }^{70} \mathrm{CNIs}$ administered after transplantation cause vasoconstriction and abnormal vascular remodeling, ${ }^{71}$ whereas steroids, ciclosporin, and tacrolimus are diabetogenic. ${ }^{72}$ Atorvastatin or pravastatin should be preferred to other statins as HMG-CoA reductase inhibitors, as they have less CYP3A and P-glycoprotein inhibitor activity. Combination treatment of statins with other lipid lowering agents, such as fibrates, may increase the risk of myotoxicity. ${ }^{73}$

\section{Cancer}

Patients with HIV are at increased risk of virus-mediated cancers such as Kaposi's sarcoma, non-Hodgkins lymphoma, human-papilloma-virus-associated cervical and anal cancers, and liver cancer. ${ }^{74,75}$ Since the advent of HAART, Kaposi's sarcoma and non-Hodgkins lymphoma rates have declined $;{ }^{76}$ however, hepatocellular carcinoma rates have increased. Although the reason for the increasing incidence of hepatocellular carcinoma is unclear, it is in part related to the increased longevity of patients with HIV co-infected with HBV or $\mathrm{HCV} .{ }^{77}$ Regular surveillance for hepatocellular carcinoma is warranted for patients with $\mathrm{HIV}$ and HCV or HBV. If the carcinoma develops, patients should be offered all conventional therapies, including liver transplantation. Human-papilloma-virus-associated cervical and anal cancers are a major concern in people with HIV, as both the risk for carcinoma and the growth of the carcinoma can be exacerbated by immunosuppression. Routine Pap smears and colonoscopies should ideally be performed in renal transplant recipients with HIV. ${ }^{78}$

\section{Conclusions}

Recognition that introduction of HAART has turned HIV into a chronic condition has been a gradual process in the transplantation community. ${ }^{79}$. Multiple studies have reported promising outcomes at 3-5 years after kidney transplantations in patients treated with HAART, ${ }^{24,27,29}$ In these patients, HIV load remains suppressed, CD4 ${ }^{+}$T-cell counts remain stable, and opportunistic infections do not seem to increase considerably. However, rates of acute organ rejections are increased in these patients, and an NIH prospective multicenter trial is currently underway exploring the long-term effects that these acute rejections have on graft survival. Co-infection with HIV and HCV is a major concern in renal transplant recipients, both in terms of treatment options and long-term effects on progression of liver disease.

In general, the management of comorbities and their increased risk is crucial in the treatment of these patients. The importance of having a team of specialists looking after renal allograft recipients with HIV cannot be overemphasized. At the very least, this team should include HIV and infectious disease specialists, pharmacists, nephrologists, transplant surgeons, primary care physicians and transplant coordinators or nephrology nurses to help manage the information flow between the patients and the caregivers. ${ }^{31,80}$ Regardless of concerns, however, evidence clearly indicates that the exclusion of patients with HIV from kidney transplantation can no longer be justified.

\section{Key points}

- Renal transplantation is both safe and effective in patients with HIV 
- Rejection rates in patients with HIV are increased, although these rejections respond to therapy

- Several interactions between highly active antiretroviral therapy drugs and immunosuppressants exist, and they should be taken into careful consideration when devising immunosuppression regimens

- Management of co-infection with HCV and HIV is challenging, with more rapid progression of liver disease in affected patients

- Treatment and patient oversight by team of specialists is critically important to the management of renal transplant recipients with HIV

\section{Box 1}

Proposed selection criteria for renal transplantation candidates with HIV-1 infection $^{62}$

\section{Exclusion criteria}

- Age $<1$ year

- Detectable HIV-1 RNA

- History of progressive multifocal leukoencephalopathy, chronic intestinal cryptosporidiosis of at least 1-month-duration, lymphoma (Burkitt's, immunoblastic or brain)

- History of multi-drug resistant fungal infection (e.g. resistant Candida krusei or Candida glabrata infection) not expected to respond to available oral antifungal agents

- History of any neoplasm except those specified in the inclusion criteria below

- Substance use as per local transplantation policy

- Advanced cardiac or pulmonary disease as per local transplantation policy

- Anatomic abnormalities precluding transplantation

- Use of interleukin-2 or granulocyte-macrophage colony-stimulating factor in the 6 months before transplantation

- Cirrhosis on liver biopsy in patients with hepatitis $\mathrm{C}$ co-infection, unless candidate is being listed for combined liver and kidney transplant

- Substantial wasting and/or malnutrition

- Concomitant conditions that, in the judgment of care providers, preclude transplantation or immunosuppression

\section{Inclusion criteria}

- Meeting standard criteria for inclusion in renal transplantation list

- $\mathrm{CD}_{4}+\mathrm{T}$-cell count $\geq 200 / \mathrm{ml}$ at any time in the 16 weeks before transplantation.

- No change in antiretroviral regimen for 3 months before transplantation

- Primary medical care provider has expertise in HIV treatment

- Ability and willingness to comply with immunosuppression protocol and antiretroviral therapy 
- Ability and willingness to undergo prophylaxis for pneumocystis pneumonia, herpes virus and fungal infection

- If hepatitis $\mathrm{C}$ co-infection is present, ability and willingness to undergo frequent post-transplantation monitoring including hepatitis $\mathrm{C}$ treatment as mandated by medical care provider and collection of liver biopsy samples

- If a history of pulmonary coccidiodomycosis exists, patient must be disease-free for at least 5 years before transplantation

- If a history of neoplasms such as cutaneous Kaposi's sarcoma, in situ anogenital carcinoma, adequately treated basal or squamous cell carcinoma of the skin or solid tumors treated with curative therapy exists, the patient must be disease-free for at least 5 years before transplantation

- If a history of renal cell carcinoma exists, patient must be disease-free for at least 2 years before transplantation

- Ability to provide informed consent. For children under the age of 7 years, only the parent can provide consent. For children aged 7-12 years, the parental or legally responsible person must provide informed consent and the minor must sign an assent. In the case of a minor between ages 13 and 18 years, the minor and parent(s) must provide informed consent

- Female candidates of child-bearing potential must have a negative serum human chorionic gonadotropin chain beta pregnancy test 14 days before transplantation. All candidates must practice barrier contraception

\section{Acknowledgments}

Drs. Frassetto and Stock receive support from NIH NIAID grant AI052748, "Solid Organ Transplantation in HIV".

\section{References}

1. Pardo V, et al. Glomerular lesions in the acquired immunodeficiency syndrome. Ann Intern Med 1984;101:429-434. [PubMed: 6476632]

2. Rao TK, et al. Associated focal and segmental glomerulosclerosis in the acquired immunodeficiency syndrome. N Engl J Med 1984;310:669-673. [PubMed: 6700641]

3. Ross MJ, Klotman PE. Recent progress in HIV-associated nephropathy. J Am Soc Nephrol 2002;13:2997-3004. [PubMed: 12444220]

4. Selik RM, Byers RH Jr, Dworkin MS. Trends in diseases reported on U.S death certificates that mentioned HIV infection 1987-1999. J Acquir Immune Defic Syndr 2002;29:378-387. [PubMed: 11917243]

5. Trullas JC, et al. Prevalence and clinical characteristics of HIV type 1-infected patients receiving dialysis in Spain: results of a Spanish survey in 2006: GESIDA 48/05 study. AIDS Res Hum Retroviruses 2008;24:1229-1235. [PubMed: 18834322]

6. Choi AI, et al. Low rates of antiretroviral therapy among HIV-infected patients with chronic kidney disease. Clin Infect Dis 2007;45:1633-1639. [PubMed: 18190326]

7. Fine DM, Perazella MA, Lucas GM, Atta MG. Renal disease in patients with HIV infection: epidemiology, pathogenesis and management. Drugs 2008;68:963-980. [PubMed: 18457462]

8. Shahinian V, et al. Prevalence of HIV-associated nephropathy in autopsies of HIV-infected patients. Am J Kidney Dis 2000;35:884-888. [PubMed: 10793023]

9. Cohen AH, Sun NC, Shapshak P, Imagawa DT. Demonstration of human immunodeficiency virus in renal epithelium in HIV-associated nephropathy. Mod Pathol 1989;2:125-128. [PubMed: 2657719] 
10. Nochy D, et al. Renal disease associated with HIV infection: a multicentric study of 60 patients from Paris hospitals. Nephrol Dial Transplant 1993;8:11-19. [PubMed: 8381928]

11. Monahan M, Tanji N, Klotman PE. HIV-associated nephropathy: an urban epidemic. Semin Nephrol 2001;21:394-402. [PubMed: 11455528]

12. Ahuja TS, et al. Is the prevalence of HIV-associated nephropathy decreasing? Am J Nephrol 1999;19:655-659. [PubMed: 10592359]

13. Winston JA, et al. Nephropathy and establishment of a renal reservoir of HIV type 1 during primary infection. N Engl J Med 2001;344:1979-1984. [PubMed: 11430327]

14. Abbott KC, Trespalacios FC, Agodoa LY, Ahuja TS. HIVAN and medication use in chronic dialysis patients in the United States: analysis of the USRDS DMMS Wave 2 study. BMC Nephrol 2003;4:5. [PubMed: 12837135]

15. Rho M, Perazella MA. Nephrotoxicity associated with antiretroviral therapy in HIV-infected patients. Curr Drug Saf 2007;2:147-154. [PubMed: 18690961]

16. Fichtenbaum CJ. Metabolic abnormalities associated with HIV infection and antiretroviral therapy. Curr Infect Dis Rep 2009;11:84-92. [PubMed: 19094829]

17. Tourret $\mathrm{J}$, et al. Outcome and prognosis factors in HIV-infected hemodialysis patients. Clin $\mathrm{J}$ Am Soc Nephrol 2006;6:1241-1247. [PubMed: 17699354]

18. Atta MG, et al. Survival during renal replacement therapy among African Americans infected with HIV type 1 in urban Baltimore, Maryland. Clin Infect Dis 2007;45:625-1632.

19. Nolan CR. Strategies for improving long-term survival in patients with ESRD. J Am Soc Nephrol 2005;16S2:S120-127. [PubMed: 16251247]

20. Stock PG, et al. Kidney and liver transplantation in human immunodeficiency virus-infected patients: a pilot safety and efficacy study. Transplantation 2003;76:370-375. [PubMed: 12883195]

21. Carter JT, Melcher ML, Carlson LL, Roland ME, Stock PG. Thymoglobulin-associated CD4+ Tcell depletion and infection risk in HIV-infected renal transplant recipients. Am J Transplant 2006;6:753-760. [PubMed: 16539632]

22. Pelletier SJ, et al. Review of transplantation in HIV patients during the HAART era. Clin Transpl 2004:63-82. [PubMed: 16704139]

23. Abbott KC, Swanson SJ, Agodoa LY, Kimmel PL. Human immunodeficiency virus infection and kidney transplantation in the era of highly active antiretroviral therapy and modern immunosuppression. J Am Soc Nephrol 2004;15:1633-1639. [PubMed: 15153575]

24. Roland ME, Stock PG. Review of solid-organ transplantation in HIV-infected patients. Transplantation 2003;75:425-429. [PubMed: 12605104]

25. Roland ME, et al. HIV-infected liver and kidney transplant recipients: 1- and 3-year outcomes. Am J Transplant 2008;8:355-365. [PubMed: 18093266]

26. Tan HP, et al. Living-related donor renal transplantation in HIV+ recipients using alemtuzumab preconditioning and steroid-free tacrolimus monotherapy: a single center preliminary experience. Transplantation 2004;78:1683-1688. [PubMed: 15591960]

27. Kumar MS, et al. Safety and success of kidney transplantation and concomitant immunosuppression in HIV-positive patients. Kidney Int 2005;67:1622-1629. [PubMed: 15780120]

28. Trullas JC, et al. Effect of thymoglobulin induction on HIV-infected renal transplant recipients: differences between HIV-positive and HIV-negative patients. AIDS Res Hum Retroviruses 2007;23:1161-1165. [PubMed: 17961099]

29. Gruber SA, et al. Preliminary experience with renal transplantation in HIV+ recipients: low acute rejection and infection rates. Transplantation 2008;86:269-274. [PubMed: 18645490]

30. Samuel D, Weber R, Stock P, Duclos-Vallee JC, Terrault N. Are HIV-infected patients candidates for liver transplantation? J Hepatol 2008;48:697-707. [PubMed: 18331763]

31. Carlson L. Clinical management of the HIV-positive kidney transplant recipient. Nephrology Nursing J 2008;35:559-567.

32. Bhagani S, Sweny P, Brook G. British HIV Association. Guidelines for kidney transplantation in patients with HIV disease. HIV Med 2006;7:133-139. [PubMed: 16494626] 
33. Rouet F, et al. CD4 percentages and total lymphocyte counts as early surrogate markers for pediatric HIV-1 infection in resource-limited settings. J Trop Pediatr 2006;52:346-354. [PubMed: 16782723]

34. O'Gorman MR, Zijenah LS. CD4 T cell measurements in the management of antiretroviral therapy--A review with an emphasis on pediatric HIV-infected patients. Cytometry B Clin Cytom 2008;74:S19-S26. [PubMed: 18228562]

35. Sehgal SN. Sirolimus: its discovery, biological properties, and mechanism of action. Transplant Proc 2003;35:S7-S14.

36. Roy J, Paquette JS, Fortin JF, Tremblay MJ. The immunosuppressant rapamycin represses human immunodeficiency virus type 1 replication. Antimicrob Agents Chemother 2002;46:3447-3455. [PubMed: 12384349]

37. Heredia A, et al. Rapamycin causes down-regulation of CCR5 and accumulation of anti-HIV betachemokines: an approach to suppress R5 strains of HIV-1. Proc Natl Acad Sci USA 2003;100:10411-10416. [PubMed: 12915736]

38. Lesens O, Deschenes M, Steben M, Belanger G, Tsoukas CM. Hepatitis C virus is related to progressive liver disease in human immunodeficiency virus-positive hemophiliacs and should be treated as an opportunistic infection. J Infect Dis 1999;179:1254-1258. [PubMed: 10191232]

39. Jain M, Chakravarti A, Verma V, Bhalla P. Seroprevalence of hepatitis viruses in patients infected with the human immunodeficiency virus. Indian J Pathol Microbiol 2009;52:17-19. [PubMed: 19136772]

40. Ragni MV, Nalesnik MA, Schillo R, Dang Q. Highly active antiretroviral therapy improves ESLDfree survival in HIV-HCV co-infection. Haemophilia 2009;15:552-528. [PubMed: 19347994]

41. Luan FL, et al. Impact of immunosuppressive regimen on survival of kidney transplant recipients with hepatitis C. Transplantation 2008;85:1601-1606. [PubMed: 18551066]

42. Locke JE, Montgomery RA, Warren DS, Subramanian A, Segev DL. Renal transplant in HIVpositive patients: long-term outcomes and risk factors for graft loss. Arch Surg 2009;144:83-86. [PubMed: 19153330]

43. Margolis D, et al. Abacavir and mycophenolic acid, an inhibitor of inosine monophosphate dehydrogenase, have profound and synergistic anti-HIV activity. J Acquir Immune Defic Syndr 1999;21:362-370. [PubMed: 10458616]

44. Kaur R, et al. A placebo-controlled pilot study of intensification of antiretroviral therapy with mycophenolate mofetil. AIDS Res Ther 2006;3:16-21. [PubMed: 16729890]

45. Oestreich KJ, Yoon H, Ahmed R, Boss JM. NFATc1 regulates PD-1 expression upon T cell activation. J Immunol 2008;181:4832-4839. [PubMed: 18802087]

46. Fruman DA, Klee CB, Bierer BE, Burakoff SJ. Calcineurin phosphatase activity in T lymphocytes is inhibited by FK 506 and cyclosporin A. Proc Natl Acad Sci USA 1992;89:3686-3690. [PubMed: 1373887]

47. Argyropoulos C, Mouzaki A. Immunosuppressive drugs in HIV disease. Curr Top Med Chem 2006;6:1769-1789. [PubMed: 17017956]

48. Stock PG, Roland ME. Evolving clinical strategies for transplantation in the HIV-positive recipient. Transplantation 2007;84:563-571. [PubMed: 17876267]

49. Wacher VJ, Wu CY, Benet LZ. Overlapping substrate specificities and tissue distribution of cytochrome P450 3A and P-glycoprotein: implications for drug delivery and activity in cancer chemotherapy. Mol Carcinog 1995;13:129-134. [PubMed: 7619215]

50. Benet LZ, Cummins CL, Wu CY. Unmasking the dynamic interplay between efflux transporters and metabolic enzymes. Int J Pharm 2004;277:3-9. [PubMed: 15158963]

51. Frassetto LA, et al. Immunosuppressant pharmacokinetics and dosing modifications in HIV-1 infected liver and kidney transplant recipients. Am J Transplant 2007;7:2816-2820. [PubMed: 17949460]

52. Jain AB, et al. Effect of coadministered lopinavir and ritonavir (Kaletra) on tacrolimus blood concentration in liver transplantation patients. Liver Transpl 2003;9:954-960. [PubMed: 12942457]

53. Jain AK, et al. The interaction between antiretroviral agents and tacrolimus in liver and kidney transplant patients. Liver Transpl 2002;8:841-845. [PubMed: 12200788] 
54. Vogel M, et al. Management of drug-to-drug interactions between cyclosporine A and the protease-inhibitor lopinavir/ritonavir in liver-transplanted HIV-infected patients. Liver Transpl 2004;10:939-944. [PubMed: 15237382]

55. Guaraldi G, et al. Pharmacokinetic interaction between Amprenavir/Ritonavir and FosAmprenavir on cyclosporine in two patients with human immunodeficiency virus infection undergoing orthotopic liver transplantation. Transplant Proc 2006;38:1138-1140. [PubMed: 16757288]

56. Niwa T, Murayama N, Emoto C, Yamazaki H. Comparison of kinetic parameters for drug oxidation rates and substrate inhibition potential mediated by cytochrome P450 3A4 and 3A5. Curr Drug Metab 2008;9:20-33. [PubMed: 18220568]

57. Guiard-Schmid JB, Poirier JM, Bonnard P, Meynard JL. Lack of interaction between atazanavir and proton pump inhibitors in $\mathrm{HIV}$-infected patients treated with ritonavir-boosted atazanavir. $\mathrm{J}$ Acquir Immune Defic Syndr 2006;41:393-394. [PubMed: 16540944]

58. Kiser JJ, Lichtenstein KA, Anderson PL, Fletcher CV. Effects of esomeprazole on the pharmacokinetics of atazanavir and fosamprenavir in a patient with human immunodeficiency virus infection. Pharmacotherapy 2006;26:511-514. [PubMed: 16553510]

59. Fulco PP, Vora UB, Bearman GM. Acid suppressive therapy and the effects on protease inhibitors. Ann Pharmacother 2006;40:1974-1983. [PubMed: 17077173]

60. Rashid A, et al. The impact of hepatitis $\mathrm{C}$ infection and antiviral therapy on clinical outcome in renal transplantation recipients. Saudi J Kidney Dis Transpl 1999;10:31-35. [PubMed: 18212411]

61. Miro JM, et al. [GESIDA/GESITRA-SEIMC, PNS and ONT consensus document on solid organ transplant (SOT) in HIV-infected patients in Spain (March, 2005).]. Enferm Infecc Microbiol Clin 2005;23:353-362. [PubMed: 15970168]

62. Transplant Study For People with HIV. 2009. onlinewww.HIVtransplant.com

63. Kovesdy CP, Kalantar-Zadeh K. Bone and mineral disorders in pre-dialysis CKD. Int Urol Nephrol 2008;40:427-440. [PubMed: 18368510]

64. Mitterbauer C, Oberbauer R. Bone disease after kidney transplantation. Transpl Int 2008;21:615624. [PubMed: 18346011]

65. Dolan SE, Carpenter S, Grinspoon S. Effects of weight, body composition, and testosterone on bone mineral density in HIV-infected women. J Acquir Immune Defic Syndr 2007;45:161-167. [PubMed: 17527091]

66. Kalyani RR, Gavini S, Dobs AS. Male hypogonadism in systemic disease. Endocrinol Metab Clin North Am 2007;36:333-348. [PubMed: 17543722]

67. Jacobson DL, Spiegelman D, Knox TK, Wilson IB. Evolution and predictors of change in total bone mineral density over time in HIV-infected men and women in the nutrition for healthy living study. J Acquir Immune Defic Syndr 2008;49:298-308. [PubMed: 18845956]

68. Pollet C, Paul SM, Morgan R. Immunizations in the HIV-infected patient. N J Med 2002;99:2331. [PubMed: 12014087]

69. Twardowski ZJ. Sodium, hypertension, and an explanation of the "lag phenomenon" in hemodialysis patients. Hemodial Int 2008;12:412-425. [PubMed: 19090863]

70. Flint OP, et al. The role of protease inhibitors in the pathogenesis of HIV-associated lipodystrophy: cellular mechanisms and clinical implications. Toxicol Pathol 2009;37:65-77. [PubMed: 19171928]

71. Bobadilla NA, Gamba G. New insights into the pathophysiology of cyclosporine nephrotoxicity: a role of aldosterone. Am J Physiol Renal Physiol 2007;293:F2-F9. [PubMed: 17429034]

72. Reynolds LR, Tannock LR. Management of new-onset diabetes mellitus after transplantation. Postgrad Med 2008;120:60-66. [PubMed: 18654070]

73. Corsini A. The safety of HMG-CoA reductase inhibitors in special populations at high cardiovascular risk. Cardiovasc Drugs Ther 2003;17:265-285. [PubMed: 14574085]

74. Goedert JJ, et al. Spectrum of AIDS-associated malignant disorders. Lancet 1998;351:1833-1839. [PubMed: 9652666]

75. Serraino D, Piselli P. Immunosuppression and Cancer Study Group. Cancer cohort consortium approach: cancer epidemiology in immunosuppressed groups. Methods Mol Biol 2009;471:409_ 419. [PubMed: 19109791] 
76. Engels EA, et al. Cancer risk in people infected with human immunodeficiency virus in the United States. Int J Cancer 2008;123:187-194. [PubMed: 18435450]

77. MacDonald DC, Nelson M, Bower M, Powles T. Hepatocellular carcinoma, human immunodeficiency virus and viral hepatitis in the HAART era. World J Gastroenterol 2008;14:1657-1663. [PubMed: 18350596]

78. Chin-Hong PV, Palefsky JM. Human papillomavirus anogenital disease in HIV-infected individuals. Dermatol Ther 2005;18:67-76. [PubMed: 15842614]

79. Mazuecos A, et al. Renal transplantation in HIV-infected patients in Spain. Nefrologia 2006;26:113-120. [PubMed: 16649432]

80. Roland ME, Carlson LL, Frassetto LA, Stock PG. Solid organ transplantation: referral, management, and outcomes in HIV-infected patients. AIDS Read 2006;16:664-668. 675-678. [PubMed: 17195325]

81. USPHS/IDSA Prevention of Opportunistic Infections Working Group. USPHS/IDSA guidelines for the preventionof opportunistic infections in person infected with Human Immunodeficiency Virus. MMWR 1999;48:10. 
Table 1

Recommended prophylaxis regimens for renal transplantation candidates with HIV

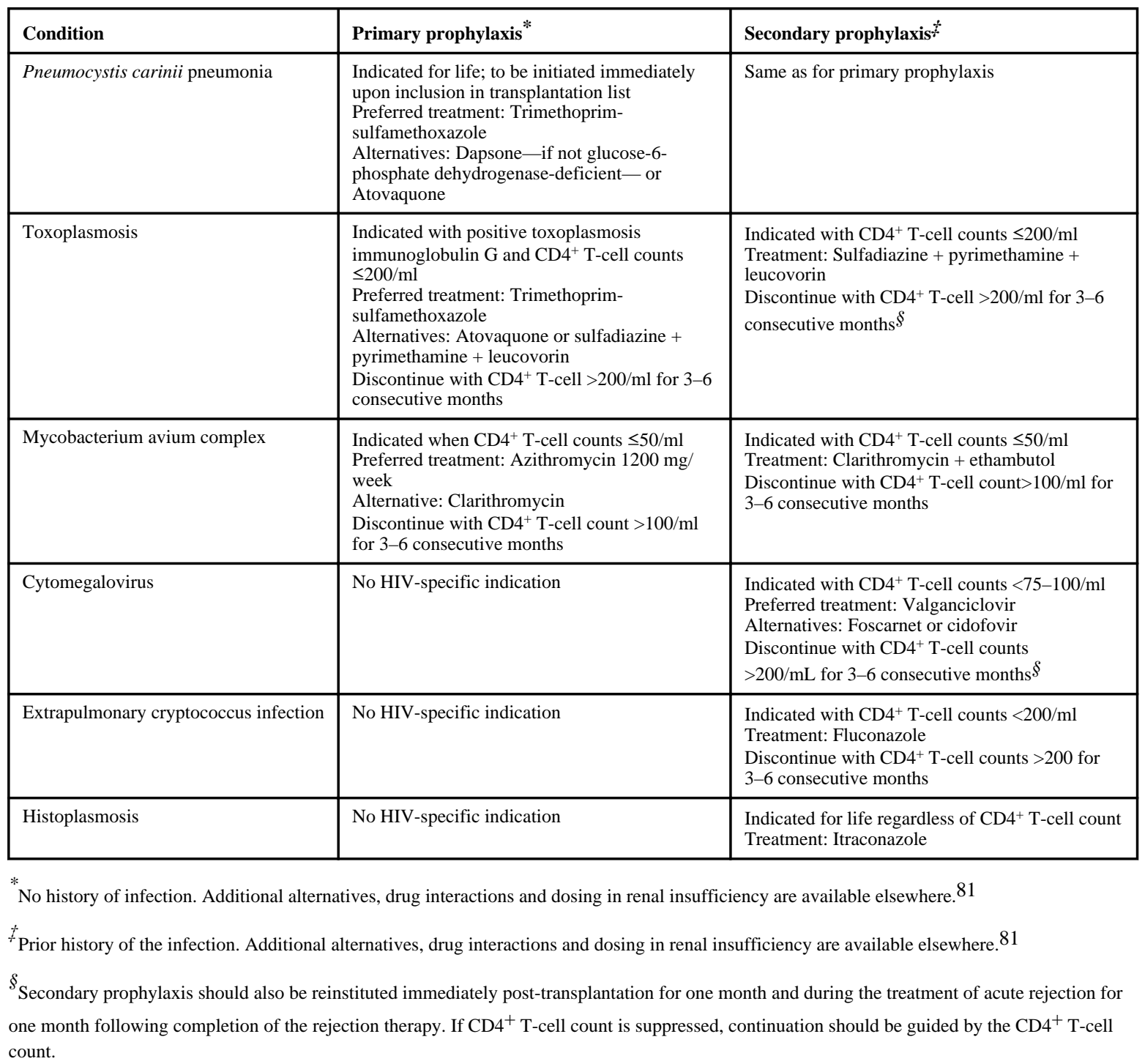

